\title{
A New Iterative Algorithm for Solving a Class of Matrix Nearness Problem
}

\author{
Xuefeng Duan ${ }^{1,2}$ and Chunmei Li ${ }^{1}$ \\ ${ }^{1}$ College of Mathematics and Computational Science, Guilin University of Electronic Technology, Guilin 541004, China \\ ${ }^{2}$ Department of Mathematics, Shanghai University, Shanghai 200444, China
}

Correspondence should be addressed to Xuefeng Duan, duanxuefeng@guet.edu.cn

Received 14 September 2011; Accepted 3 October 2011

Academic Editors: T. Allahviranloo and K. Eom

Copyright () 2012 X. Duan and C. Li. This is an open access article distributed under the Creative Commons Attribution License, which permits unrestricted use, distribution, and reproduction in any medium, provided the original work is properly cited.

\begin{abstract}
Based on the alternating projection algorithm, which was proposed by Von Neumann to treat the problem of finding the projection of a given point onto the intersection of two closed subspaces, we propose a new iterative algorithm to solve the matrix nearness problem associated with the matrix equations $A X B=E, C X D=F$, which arises frequently in experimental design. If we choose the initial iterative matrix $X_{0}=0$, the least Frobenius norm solution of these matrix equations is obtained. Numerical examples show that the new algorithm is feasible and effective.
\end{abstract}

\section{Introduction}

Denoted by $R^{m \times n}$ be the set of $m \times n$ real matrices, $A^{T}$ and $A^{+}$be the transpose and Moore-Penrose generalized inverse of the matrix $A$, respectively. For $A, B \in R^{m \times n},\langle A, B\rangle=$ trace $\left(B^{T} A\right)$ denotes the inner product of the matrix $A$ and $B$. The induced norm is the so-called Frobenius norm, that is, $\|A\|=\langle A, A\rangle^{1 / 2}$; then $R^{m \times n}$ is a real Hilbert space. Let $M$ be a closed convex subset in a real Hilbert space $H$ and $u$ be a point in $H$; then the point in $M$ nearest to $u$ is called the projection of $u$ onto $M$ and denoted by $P_{M}(u)$; that is to say, $P_{M}(u)$ is the solution of the following minimization problem (see $[1,2])$

$$
\min _{x \in M}\|x-u\|
$$

that is,

$$
\left\|P_{M}(u)-u\right\|=\min _{x \in M}\|x-u\| .
$$

The problem of finding a nearness matrix $\hat{X}$ in a constraint matrix set to a given matrix $\bar{X}$ is called the matrix nearness problem. Because the preliminary estimation $\bar{X}$ is frequently obtained from experiments, it may not satisfy the given restrictions. Hence it is necessary to find a nearness matrix $\hat{X}$ in this constraint matrix set to replace the estimation $\bar{X}[3]$. In the area of structure design, finite element model updating and control theory, and so forth, the matrix set is always the (constraint) solution set or the least square (constraint) solution set of some matrix equations [4-6]. Thus, the problem mentioned above is also called the matrix nearness problem associated with the matrix equation. Recently, there are many discussions on the matrix nearness problem associated with some matrix equations. For instance, see [4, 6-14].

In this paper, we consider the following problem.

Problem 1. Given matrices $A \in R^{p \times n}, B \in R^{n \times q}, C \in$ $R^{s \times n}, D \in R^{n \times t}, E \in R^{p \times q}, F \in R^{s \times t}$, and $\bar{X} \in R^{n \times n}$, find $\hat{X} \in \Omega$ such that

$$
\|\hat{X}-\bar{X}\|=\min _{X \in \Omega}\|X-\bar{X}\|,
$$

where

$$
\Omega=\left\{X \in R^{n \times n} \mid A X B=E, C X D=F\right\} .
$$

Obviously, $\Omega$ is the solution set of the matrix equations

$$
A X B=E, \quad C X D=F,
$$

and $\hat{X}$ is the optimal approximate solution of (5) to the given matrix $\bar{X}$. In particular, if $\bar{X}=0$, then the solution $\hat{X}$ of 
Problem 1 is just the least Frobenius norm solution of the matrix equations (5). It is easy to verify that $\Omega$ is convex set; then the solution of Problem 1 is unique.

The matrix equations (5) and its matrix nearness problem have been extensively studied for the past 40 or more years. Wang [15] and Navarra et al. [16] gave some conditions for the existence of a solution and some representations of the general common solution to (5). By the projection theorem and matrix decompositions, Liao et al. [6] gave an analytical expression of the optimal approximate least square symmetric solution of (5). However, these direct methods may be less efficient for the large sparse coefficient matrices due to the limited storages and the speeds of the computers. Therefore, iterative methods for solving the matrix equations (5) have attracted much interests recently. Peng et al. [11] and Chen et al. [7] proposed some iterative methods to compute the symmetric solutions and optimal approximate symmetric solution of (5). An efficient iterative method was presented to solve the matrix nearness Problem 1 associated with the matrix equations (5) in [13]. Ding et al. [17] considered the unique solution of the matrix equations (5) and used gradient-based iterative algorithm to compute the unique solution. The (least square) solution and the optimal approximate (least square) solution of (5), which is constrained as bisymmetric, reflexive, generalized reflexive, generalized centrosymmetric, were studied in [7-10, 12].

The alternating projection algorithm dates back to von Neumann [18], who treated the problem of finding the projection of a given point onto the intersection of two closed subspaces. Later, Bauschke and Borwein [1] extended the analysis of Von Neumann's alternating projection scheme to the case of two closed affine subspaces . There are many variations and extensions of the alternating projection algorithm, and we can use them to find the projection of a given point onto the intersection of $k(k \geq 2)$ closed subspaces [19] and $k(k \geq 2)$ closed convex sets $[20,21]$. For a complete discussion on the alternation projection algorithm see Deutsch [2].

In this paper, we propose a new algorithm to solve Problem 1. We state Problem 1 as the minimization of a convex quadratic function over the intersection of two closed affine subspaces in the vector space $R^{n \times n}$. From this point of view, Problem 1 can be solved by the alternating projection algorithm. If we choose the initial iterative matrix $X_{0}=0$, the least Frobenius norm solution of the matrix equations $A X B=E, C X D=F$ is obtained. In the end, we use some numerical examples to show that the new algorithm is faster and lower computational cost for each step than the algorithm proposed by Sheng and Chen [13] to solve Problem 1. Especially, the CPU time and iteration steps of our algorithm increase slowly as the dimension of the matrix is increasing; so our algorithm is suitable for large-scale problems.

\section{Alternating Projection Algorithm for Solving Problem 1}

In this section, we apply the alternating projection algorithm to solve Problem 1. We begin with two lemmas.
Lemma 1 (see [1, Theorem 4.1]). Let $M_{1}=a+\widetilde{M}_{1}, M_{2}=$ $b+\widetilde{M}_{2}$ be closed affine subspaces in a Hilbert space $H$ and $u$ be a point in $H$. Here, $\widetilde{M}_{1}$ and $\widetilde{M}_{2}$ are closed subspaces and $a, b \in H$. If $M_{1} \cap M_{2} \neq \varnothing$, then the sequences $\left\{x_{k}\right\}$ and $\left\{y_{k}\right\}$ generated by the alternating projection algorithm

$$
\begin{gathered}
x_{0}=u, \quad y_{k+1}=P_{M_{1}}\left(x_{k}\right), \\
x_{k+1}=P_{M_{2}}\left(y_{k+1}\right), \quad k=0,1,2, \ldots
\end{gathered}
$$

both converge to the projection of the point $u$ onto the intersection of $M_{1}$ and $M_{2}$, that is,

$$
x_{k} \longrightarrow P_{M_{1} \cap M_{2}}(u), \quad y_{k} \longrightarrow P_{M_{1} \cap M_{2}}(u), \quad k \longrightarrow+\infty \text {. }
$$

Lemma 2 (see [22, Theorem 9.3.1]). Let $A \in R^{p \times n}, B \in$ $R^{n \times q}$ and $E \in R^{p \times q}$, be known matrices. Then the matrix equation $A X B=E$ has a solution if and only if

$$
A A^{+} E B^{+} B=E,
$$

and the representation of the solution is

$$
X=A^{+} E B^{+}+Y-A^{+} A Y B B^{+},
$$

where $Y \in R^{n \times n}$ is arbitrary.

Lemma 3 (see [22, Theorem 9.3.2]). Given $Z \in R^{n \times n}$, set

$\Re=\left\{X \in R^{n \times n} \mid A X B=E, A \in R^{p \times n}, B \in R^{n \times q}, E \in R^{p \times q}\right\}$,

then the solution $\hat{X}$ of the following problem

$$
\min _{X \in \mathfrak{R}}\|X-Z\|
$$

is

$$
\hat{X}=Z+A^{+}(E-A Z B) B^{+}
$$

that is,

$$
\|\hat{X}-Z\|=\min _{X \in \mathfrak{R}}\|X-Z\|
$$

Now we begin to use the alternating projection algorithm (6) to solve Problem 1. Firstly, we define two sets

$$
\begin{aligned}
& \Omega_{1}=\left\{X \in R^{n \times n} \mid A X B=E\right\}, \\
& \Omega_{2}=\left\{X \in R^{n \times n} \mid C X D=F\right\} .
\end{aligned}
$$

It is easy to know that $\Omega=\Omega_{1} \cap \Omega_{2}$, and if the set $\Omega$ is nonempty, then

$$
\Omega=\Omega_{1} \cap \Omega_{2} \neq \varnothing .
$$


And by Lemma 2, the sets $\Omega_{1}$ and $\Omega_{2}$ can be equivalently written as

$$
\begin{aligned}
\Omega_{1} & =\{X \mid A X B=E\} \\
& =\left\{X=A^{+} E B^{+}+Y-A^{+} A Y B B^{+} \mid Y \in R^{n \times n}\right\} \\
& =A^{+} E B^{+}+\left\{Y-A^{+} A Y B B^{+} \mid Y \in R^{n \times n}\right\}, \\
\Omega_{2} & =\{X \mid C X D=F\} \\
& =\left\{X=C^{+} F D^{+}+Y-C^{+} C Y D D^{+} \mid Y \in R^{n \times n}\right\} \\
& =C^{+} F D^{+}+\left\{Y-C^{+} C Y D D^{+} \mid Y \in R^{n \times n}\right\} .
\end{aligned}
$$

Hence, $\Omega_{1}$ and $\Omega_{2}$ are closed affine subspaces.

After defining the sets $\Omega_{1}$ and $\Omega_{2}$, Problem 1 can be rewritten as finding $\hat{X} \in \Omega=\Omega_{1} \cap \Omega_{2}$, such that

$$
\|\hat{X}-\bar{X}\|=\min _{X \in \Omega_{1} \cap \Omega_{2}}\|X-\bar{X}\| .
$$

Noting the equalities (17) and (2), it is easy to find that

$$
\hat{X}=P_{\Omega_{1} \cap \Omega_{2}}(\bar{X}) .
$$

Therefore, Problem 1 can be converted equivalently into finding the projection $P_{\Omega_{1} \cap \Omega_{2}}(\bar{X})$ of a given matrix $\bar{X}$ onto the intersection set $\Omega_{1} \cap \Omega_{2}$. Now we will use alternating projection algorithm (6) to compute the projection $P_{\Omega_{1} \cap \Omega_{2}}(\bar{X})$. Consequently, we can get the solution $\hat{X}$ of Problem 1 .

By (6) we can see that the key problems to realize the alternating projection algorithm (6) are how to compute the projections $P_{\Omega_{1}}(Z), P_{\Omega_{2}}(Z)$ of a matrix $Z$ onto $\Omega_{1}$ and $\Omega_{2}$, respectively. Such problems are perfectly solvable in the following theorems.

Theorem 1. Suppose that the set $\Omega_{1}$ is nonempty. For a given $n \times n$ matrix $Z$, we have

$$
P_{\Omega_{1}}(Z)=Z+A^{+}(E-A Z B) B^{+} .
$$

Proof. By (1) and (2), we know that the projection $P_{\Omega_{1}}(Z)$ is the solution of the following minimization problem

$$
\min _{X \in \Omega_{1}}\|X-Z\|
$$

and according to Lemma 3 we know that the solution of the minimization problem (20) is $Z+A^{+}(E-A Z B) B^{+}$. Hence,

$$
P_{\Omega_{1}}(Z)=Z+A^{+}(E-A Z B) B^{+} .
$$

Theorem 2. Suppose that the set $\Omega_{2}$ is nonempty. For a given $n \times n$ matrix $Z$, we have

$$
P_{\Omega_{2}}(Z)=Z+C^{+}(F-C Z D) D^{+} .
$$

Proof. The proof is similar to that of Theorem 1 and is omitted here.

By the alternation projection algorithm (6) and Theorems 1 and 2, we get a new algorithm to solve Problem 1 which can be stated as follows.
Algorithm 1. One has

(1) set $\widetilde{A}=A^{+}, \widetilde{B}=B^{+}, \widetilde{C}=C^{+}, \widetilde{D}=D^{+}$;

(2) $\operatorname{set} X_{0}=\bar{X}$;

(3) for $k=0,1,2,3, \ldots$

$$
\begin{gathered}
Y_{k+1}=P_{\Omega_{1}}\left(X_{k}\right)=X_{k}+\widetilde{A}\left(E-A X_{k} B\right) \widetilde{B}, \\
X_{k+1}=P_{\Omega_{2}}\left(Y_{k+1}\right)=Y_{k+1}+\widetilde{C}\left(F-C Y_{k+1} D\right) \tilde{D},
\end{gathered}
$$

end.

By Lemma 1 and (15) and (16), we get the convergence theorem for Algorithm 1.

Theorem 3. If the set $\Omega$ is nonempty, then the matrix sequences $\left\{X_{k}\right\}$ and $\left\{Y_{k}\right\}$ generated by Algorithm 1 both converge to the projection $P_{\Omega_{1} \cap \Omega_{2}}(\bar{X})$ of $\bar{X}$ onto the intersection of $\Omega_{1}$ and $\Omega_{2}$, that is,

$$
X_{k} \longrightarrow P_{\Omega_{1} \cap \Omega_{2}}(\bar{X}), \quad Y_{k} \longrightarrow P_{\Omega_{1} \cap \Omega_{2}}(\bar{X}), \quad k \longrightarrow+\infty .
$$

Proof. If the set $\Omega$ is nonempty, by (15) we have

$$
\Omega_{1} \cap \Omega_{2} \neq \varnothing \text {. }
$$

And noting (16), we know that the sets $\Omega_{1}$ and $\Omega_{2}$ are closed affine subspaces in Hilbert space $R^{m \times n}$. Hence, by Lemma 1 we derive that the matrix sequences $\left\{X_{k}\right\}$ and $\left\{Y_{\mathrm{k}}\right\}$ generated by Algorithm 1 both converge to the projection $P_{\Omega_{1} \cap \Omega_{2}}(\bar{X})$ of $\bar{X}$ onto the intersection of $\Omega_{1}$ and $\Omega_{2}$, that is,

$$
X_{k} \longrightarrow P_{\Omega_{1} \cap \Omega_{2}}(\bar{X}), \quad Y_{k} \longrightarrow P_{\Omega_{1} \cap \Omega_{2}}(\bar{X}), \quad k \longrightarrow+\infty \text {. }
$$

Combining Theorem 3 and the equalities (18) and (17), we have the following.

Theorem 4. If the set $\Omega$ is nonempty, then the matrix sequence $\left\{X_{k}\right\}$ and $\left\{Y_{k}\right\}$ generated by Algorithm 1 both converge to the unique solution of Problem 1. Moreover, if the initial matrix $X_{0}=\bar{X}=0$, then the matrix sequence $\left\{X_{k}\right\}$ and $\left\{Y_{k}\right\}$ both converge to the least Frobenius norm solution of the matrix equations $A X B=E, C X D=F$.

\section{Numerical Experiments}

In this section, we give some numerical examples to illustrate that the new algorithm is feasible and effective to solve Problem 1. All programs are written in MATLAB 7.8. We denote

$$
\text { Error }=\|E-A X B\|+\|F-C X D\|,
$$

and use the practical stopping criterion Error $\leq 1.0 \times 10^{-10}$. 
Example 1. Consider Problem 1 with

$$
\begin{aligned}
& A=\left(\begin{array}{ccccc}
1 & 3 & 1 & 3 & 1 \\
3 & -7 & 3 & -7 & 3 \\
11 & 6 & 11 & 6 & 11 \\
-5 & 5 & -5 & 5 & -5 \\
9 & 4 & 9 & 4 & 9 \\
1 & 3 & 1 & 3 & 1
\end{array}\right), \\
& B=\left(\begin{array}{ccccc}
-1 & 4 & -1 & 4 & -1 \\
5 & -1 & 5 & -1 & 5 \\
-1 & -2 & -1 & -2 & -1 \\
3 & 9 & 3 & 9 & 3 \\
7 & -8 & 7 & -8 & 7
\end{array}\right) \text {, } \\
& E=\left(\begin{array}{ccccc}
117 & 18 & 117 & 18 & 117 \\
-65 & -10 & -65 & -10 & -65 \\
585 & 90 & 585 & 90 & 585 \\
-65 & -10 & -65 & -10 & -65 \\
455 & 70 & 455 & 70 & 455 \\
117 & 18 & 117 & 18 & 117
\end{array}\right), \\
& C=\left(\begin{array}{ccccc}
3 & -5 & 3 & -5 & 7 \\
-4 & 3 & -2 & 9 & 5 \\
3 & -5 & 3 & -5 & 7
\end{array}\right) \text {, } \\
& D=\left(\begin{array}{cccccc}
5 & 4 & -1 & 5 & 1 & 4 \\
-2 & 3 & 5 & -2 & 5 & 3 \\
3 & 5 & -1 & 3 & -1 & 5 \\
3 & -6 & 3 & 2 & 3 & -6 \\
1 & 19 & 7 & 1 & 7 & 19
\end{array}\right), \\
& F=\left(\begin{array}{cccccc}
30 & 75 & 39 & 27 & 45 & 75 \\
110 & 275 & 143 & 99 & 165 & 275 \\
30 & 75 & 39 & 27 & 45 & 75
\end{array}\right)
\end{aligned}
$$

Here we use ones $(n)$ and $\operatorname{zeros}(n)$ to stand for $n \times n$ matrix of ones and zeros. It is easy to verify that $X=$ ones(5) is a solution of the matrix equations (5); that is to say, the set $\Omega$ is nonempty. Therefore we can use Algorithm 1 to solve Problem 1.

Let $X_{0}=\bar{X}=\operatorname{zeros}(5)$. After 5 iterations of Algorithm 1, we get the optimal approximate solution

$$
\hat{X} \approx X_{5}=\left(\begin{array}{ccccc}
-0.6817 & 0.7813 & -0.5503 & 0.9637 & 0.7808 \\
0.0152 & 0.8185 & -0.2866 & 0.9699 & 0.8181 \\
0.0113 & 0.8178 & -0.2917 & 0.9698 & 0.8173 \\
0.6091 & 0.9280 & 0.4893 & 0.9980 & 0.9278 \\
0.9497 & 0.9907 & 0.9342 & 0.9985 & 0.9907
\end{array}\right) \text {, }
$$

which is also the least Frobenius norm solution of the matrix equations (5), and its residual error

$$
\text { Error } \approx\left\|E-A X_{5} B\right\|+\left\|F-C X_{5} D\right\|=2.78 \times 10^{-11} .
$$

By concrete computations, we know that the distance from $\bar{X}$ to the solution set $\Omega$ is

$$
\min _{X \in \Omega}\|X-\bar{X}\|=\|\hat{X}-\bar{X}\| \approx\left\|X_{5}-\bar{X}\right\|=3.9057
$$

Let $X_{0}=\bar{X}=$ ones(5). After 6 iterations of Algorithm 1, we get the optimal approximate solution

$$
\hat{X} \approx X_{6}=\left(\begin{array}{ccccc}
5.7467 & 1.8747 & 7.2013 & 1.1452 & 1.8770 \\
4.9392 & 1.7259 & 6.1463 & 1.1205 & 1.7278 \\
4.9548 & 1.7288 & 6.1668 & 1.1209 & 1.7307 \\
2.5636 & 1.2881 & 3.0427 & 1.478 & 1.2889 \\
1.2013 & 1.0371 & 1.2630 & 1.0062 & 1.372
\end{array}\right)
$$

and its residual error

$$
\text { Error } \approx\left\|E-A X_{6} B\right\|+\left\|F-C X_{6} D\right\|=3.89 \times 10^{-11} .
$$

By concrete computations, we know that the distance from $\bar{X}$ to the solution set $\Omega$ is

$$
\min _{X \in \Omega}\|X-\bar{X}\|=\|\hat{X}-\bar{X}\| \approx\left\|X_{6}-\bar{X}\right\|=16.0902 .
$$

Example 1 shows that Algorithm 1 is feasible to solve Problem 1.

Example 2. Consider Problem 1 with

$$
\begin{gathered}
A=\operatorname{rand}(100, n), \quad B=\operatorname{rand}(n, 150), \\
E=A * \operatorname{ones}(n) * B, \quad C=\operatorname{rand}(70, n), \\
D=\operatorname{rand}(n, 120), \quad F=C * \operatorname{ones}(n) * D,
\end{gathered}
$$

where $\operatorname{rand}(s, t)$ stand for $s \times t$ random matrix. Let the given matrix $\bar{X}=\operatorname{zeros}(n)$. It is easy to verify that $X=$ ones $(n)$ is the solution of the matrix equations (5); that is, the set $\Omega$ is nonempty; therefore, we can use Algorithm 1 and the following algorithm proposed by Sheng and Chen [13] to solve Problem 1.

Algorithm 2. One has

(1) input $A, B, C, D, E, F$ and $X_{0}$;

(2) calculate

$$
\begin{gathered}
R_{0}=E-A X_{0} B, \\
r_{0}=F-C X_{0} D, \\
P_{0}=A^{T} R_{0} B^{T}, \\
Q_{0}=C^{T} r_{0} D^{T} ;
\end{gathered}
$$

(3) if $R_{k}=0, r_{0}=0$, then stop; else, $k:=k+1$; 
TABLE 1

\begin{tabular}{cccc}
\hline \multirow{5}{*}{$n=50$} & & Algorithm 1 & Algorithm 2 \\
\hline \multirow{5}{*}{$n=80$} & IT & 5 & 36 \\
& CPU & 0.019262 & 0.125212 \\
& ERR & $3.46 \times 10^{-11}$ & $9.01 \times 10^{-10}$ \\
& DIS & 50.00 & 50.00 \\
\hline \multirow{5}{*}{$n=100$} & IT & 8 & 167 \\
& CPU & 0.096335 & 4.660955 \\
& ERR & $2.31 \times 10^{-10}$ & $9.26 \times 10^{-10}$ \\
& MIN & 80.00 & 80.0010 \\
\hline \multirow{5}{*}{$n=150$} & IT & 16 & 1673 \\
& CPU & 0.168511 & 17.604961 \\
& ERR & $5.21 \times 10^{-10}$ & $9.8 \times 10^{-10}$ \\
& MIN & 99.9997 & 100.0001 \\
\hline & IT & 79 & - \\
& CPU & 2.589103 & - \\
\hline
\end{tabular}

(4) calculate

$$
\begin{gathered}
X_{k}=X_{k-1}+\frac{\left\|R_{k-1}\right\|^{2}+\left\|r_{k-1}\right\|^{2}}{\left\|P_{k-1}+Q_{k-1}\right\|^{2}}\left(P_{k-1}+Q_{k-1}\right), \\
R_{k}=E-A X_{k} B, \\
r_{k}=F-C X_{k} D, \\
P_{k}=A^{T} R_{k} B^{T}+\frac{\left\|R_{k}\right\|^{2}+\left\|r_{k}\right\|^{2}}{\left\|R_{k-1}\right\|^{2}+\left\|r_{k-1}\right\|^{2}}, \\
Q_{k}=C^{T} r_{k} D^{T}+\frac{\left\|R_{k}\right\|^{2}+\left\|r_{k}\right\|^{2}}{\left\|R_{k-1}\right\|^{2}+\left\|r_{k-1}\right\|^{2}} Q_{k-1} ;
\end{gathered}
$$

(5) go to step 3.

It is easy to see that Algorithm 1 has lower computational cost for each step in the comparison with Algorithm 2. Experiments show that Algorithm 1 and Algorithm 2 are feasible to solve Problem 1. We list the iteration steps (denoted by IT), CPU time (denoted by CPU), residual error (denoted by ERR), and the distance $\left\|X_{\mathrm{IT}}-\bar{X}\right\|$ (denoted by DIS) in Table 1.

From Table 1, we can see that Algorithm 1 outperforms Algorithm 2 in iteration step and CPU time. Therefore our algorithm is faster than the algorithm proposed by Sheng and Chen [13]. Especially, the CPU time and iteration steps of our algorithm increase slowly as the dimension $n$ is increasing; so our algorithm is suitable for large-scale problems.

\section{Conclusion}

The alternating projection algorithm dates back to von Neumann [18], who treated the problem of finding the projection of a given point onto the intersection of two closed subspace. In this paper, we first apply the alternating projection algorithm to solve Problem 1, which occurs frequently in experimental design [23]. If we choose the initial matrix $X_{0}=0$, the least Frobenius norm solution of the matrix equations $A X B=E, C X D=F$ can be obtained. Numerical examples show that the new algorithm is faster and lower computational cost for each step than the algorithm proposed by Sheng and Chen [13] to solve Problem 1. Especially, the CPU time and iteration steps of the new algorithm increase slowly as the matrix's dimension is increasing; so the alternating projection algorithm is suitable for large-scale matrix nearness problems.

\section{Acknowledgments}

The work was supported in part by National Science Foundation of China (11101100; 10861005) and Natural Science Foundation of Guangxi Province (0991238; 2011GXNSFA018138).

\section{References}

[1] H. H. Bauschke and J. M. Borwein, "Dykstra's alternating projection algorithm for two sets," Journal of Approximation Theory, vol. 79, no. 3, pp. 418-443, 1994.

[2] F. Deutsch, Best Approximation in Inner Product Spaces, Springer, New York, NY, USA, 2001.

[3] N. Higham, Matrix Nearness Problems and Applications, Oxford University Press, London, UK, 1989.

[4] H. Dai and P. Lancaster, "Linear matrix equations from an inverse problem of vibration theory," Linear Algebra and Its Applications, vol. 246, pp. 31-47, 1996.

[5] M. Friswell and J. Mottorshead, Finite Element Model Updating in Structure Dynamics, Kluwer Academic publisher, Dodrecht, The Netherlands, 1995.

[6] A. P. Liao, Y. Lei, and S. F. Yuan, "The matrix nearness problem for symmetric matrices associated with the matrix equation $\left[A^{T} X A, B^{T} X B\right]=[C, D]$," Linear Algebra and Its Applications, vol. 418, no. 2-3, pp. 939-954, 2006.

[7] Y. Chen, Z. Peng, and T. Zhou, "LSQR iterative common symmetric solutions to matrix equations $A X B=E$ and $C X D=F$,' Applied Mathematics and Computation, vol. 217, no. 1, pp. 230-236, 2010.

[8] J. Cai and G. Chen, "An iterative algorithm for the least squares bisymmetric solutions of the matrix equations $A_{1} X B_{1}=C_{1}$, $A_{2} \mathrm{XB}_{2}=C_{2}$," Mathematical and Computer Modelling, vol. 50, no. 7-8, pp. 1237-1244, 2009.

[9] M. Dehghan and M. Hajarian, "An iterative algorithm for solving a pair of matrix equations $A X B=E$ and $C X D=F$ over generalized centro-symmetric matrices," Computers and Mathematics with Applications, vol. 56, no. 12, pp. 3246-3260, 2008.

[10] L. Fanliang, H. Xiyan, and Z. Lei, "The generalized reflexive solution for a class of matrix equations $(A X=B, X C=D)$," Acta Mathematica Scientia, vol. 28, no. 1, pp. 185-193, 2008.

[11] Y. X. Peng, X. Y. Hu, and L. Zhang, "An iterative method for symmetric solutions and optimal approximation solution of the system of matrix equations $A_{1} X B_{1}=C_{1}, A_{2} X B_{2}=C_{2}$," Applied Mathematics and Computation, vol. 183, no. 2, pp. 1127-1137, 2006.

[12] Z. H. Peng, X. Y. Hu, and L. Zhang, "An efficient algorithm for the least-squares reflexive solution of the matrix equation 
$A_{1} X B_{1}=C_{1}, A_{2} X B_{2}=C_{2}, "$ Applied Mathematics and Computation, vol. 181, no. 2, pp. 988-999, 2006.

[13] X. Sheng and G. Chen, "A finite iterative method for solving a pair of linear matrix equations $(A X B, C X D)=(C X D)$," Applied Mathematics and Computation, vol. 189, no. 2, pp. 1350-1358, 2007.

[14] S. Yuan, A. Liao, and G. Yao, "The matrix nearness problem associated with the quaternion matrix equation $A^{T} X A+$ $B^{T} X B=C$," Journal of Applied Mathematics and Computing, vol. 37, no. 1-2, pp. 133-144, 2011.

[15] Q. W. Wang, "A system of matrix equations and a linear matrix equation over arbitrary regular rings with identity," Linear Algebra and Its Applications, vol. 384, no. 1-3, pp. 43-54, 2004.

[16] A. Navarra, P. L. Odell, and D. M. Young, "Representation of the general common solution to the matrix equations $A_{1} X B_{1}=C_{1}, A_{2} X B_{2}=C_{2}$ with applications," Computers and Mathematics with Applications, vol. 41, no. 7-8, pp. 929-935, 2001.

[17] J. Ding, Y. Liu, and F. Ding, "Iterative solutions to matrix equations of the form $A_{i} X B_{i}=F_{i}$," Computers and Mathematics with Applications, vol. 59, no. 11, pp. 3500-3507, 2010.

[18] J. von Numann, Functional Operators, vol. 11, Princeton University Press, Princeton, NJ, USA, 1950.

[19] I. Halperin, "The product of projection operators," Acta Scientiarum Mathematicarum, vol. 23, pp. 96-99, 1962.

[20] R.L. Dykstra, "An algorithm for restricted least-squares regression," Journal of the American Statistical Association, vol. 78, pp. 837-842, 1983.

[21] R. Escalante and M. Raydan, "Dykstra's algorithm for a constrained least-squares matrix problem," Numerical Linear Algebra with Applications, vol. 3, no. 6, pp. 459-471, 1996.

[22] H. Dai, Matrix Theory, Science Press, Beijing, China, 2001.

[23] T. Meng, "Experimental design and decision support," in Expert system the Technology of Knowledge Management and Decision Making for 21st Centry, C. Leondes, Ed., vol. 1, Academic Press, New York, NY, USA, 2001. 


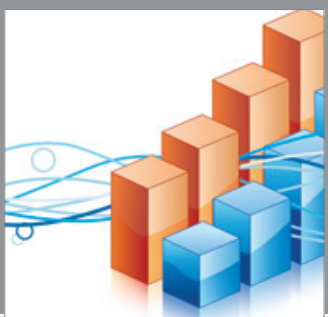

Advances in

Operations Research

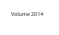

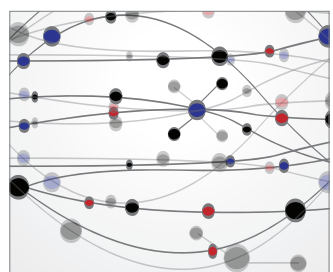

\section{The Scientific} World Journal
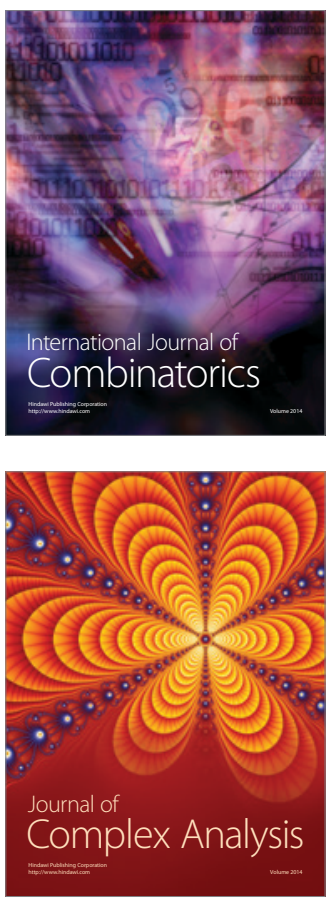

International Journal of

Mathematics and

Mathematical

Sciences
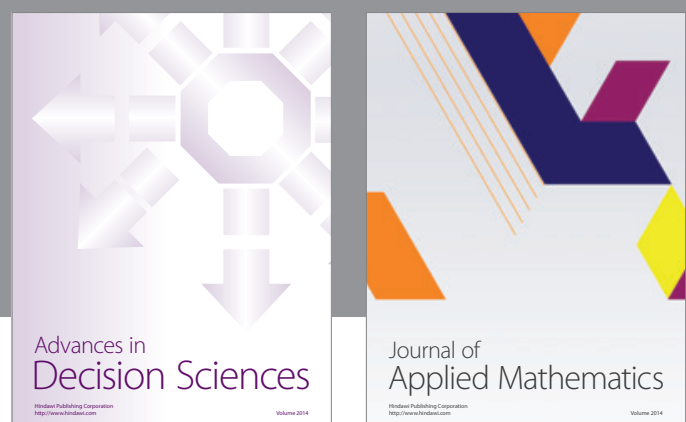

Journal of

Applied Mathematics
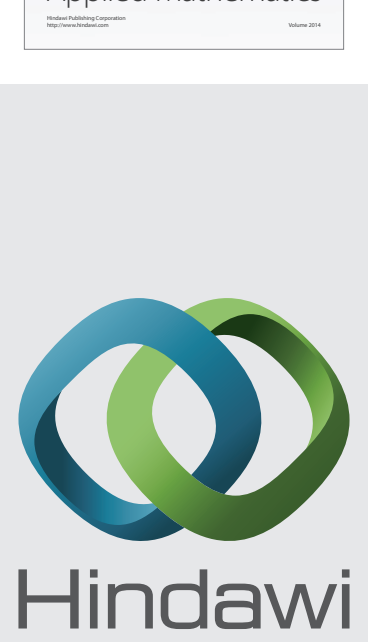

Submit your manuscripts at http://www.hindawi.com
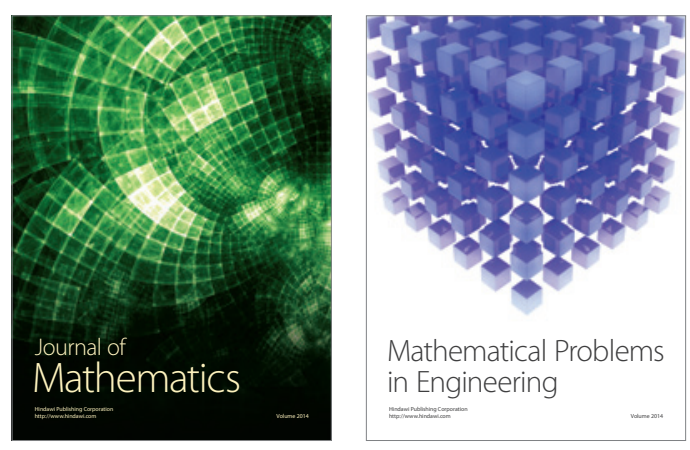

Mathematical Problems in Engineering
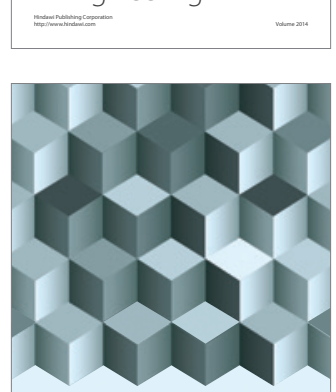

Journal of

Function Spaces
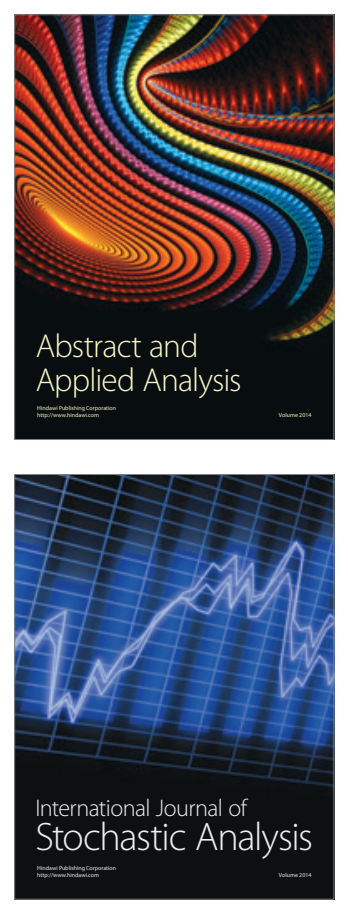

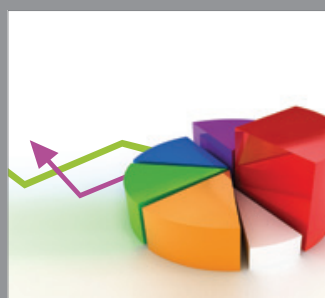

ournal of

Probability and Statistics

Promensencen
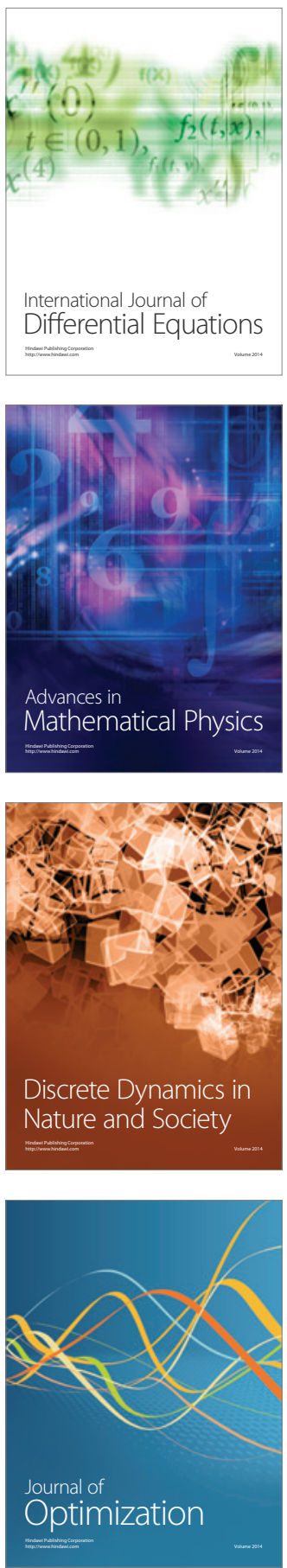